\title{
Ultrafast photonic crystal nanocavity lasers and optical switches.
}

\author{
Ilya Fushman ${ }^{a}$. Dirk Englund ${ }^{a}$. Hatice Altug ${ }^{b}$. Bryan Ellis ${ }^{a}$. Andrei Faraon ${ }^{a}$. and Jelena \\ Vučković ${ }^{a}$ \\ ${ }^{a}$ Department of Applied Physics. Stanford University: Stanford. California 94305. USA \\ ${ }^{b}$ Electrical and Computer Engineering Department, Boston University, Boston, Massachusetts \\ 02215, USA
}

\begin{abstract}
We have recently demonstrated an ultrafast photonic crvstal laser and cavity coupled laser array with modulation rates of $1 \mathrm{THz}$ at room temperature, a $20 \mathrm{GHz}$ optical modulator with activation energies of $60 \mathrm{fJ}$ and a quantum dot photonic crystal laser with large signal modulation rates of $30 \mathrm{GHz}$. These devices are enabled by the enhanced light-matter interaction in photonic crystals, and serve as the building blocks of on-optical information processing circuits.
\end{abstract}

Keywords: photonic crystal, optical communications, laser, modulator, quantum well, quantum dot, optical nonlinearity

\section{INTRODUCTION}

The miniaturization and improvement in performance of optical components, such as lasers, switches and modulators will drive the next revolution in optical telecommunication and optical interconnects, and permeate fields related to metrology and lab-on-a-chip biological experiments. ${ }^{1-3}$ Photonic crystals (PC) represent an almost ideal platform for realizing optoelectronic circuits: they are lithographically defined in any high index material, and the device performance is thus independent of the choice of substrate, as long as a high index contrast is provided. Tuning of the device geometry allows full control over the operational wavelength and propagation of light in these materials. Furthermore, PC circuits are planar, and thus devices can be monolithically integrated in a single step, circumventing cumbersome and expensive flip-chip bonding techniques. Finally, the PC devices take advantage of new regimes of light matter interaction, as PC cavities with extremely small optical volumes and large $\mathrm{Q}$-factors can be used to modify the radiative properties of light emitters embedded in them and to speed up processes of light absorption and generation. ${ }^{4.5}$ This property has led to the development of ultra-low threshold $(<1 \mu \mathrm{W})$, ultra-fast $(>\mathrm{THz})$ lasers and optical switches. ${ }^{6-10}$ The control over light matter interaction follows from the inherently single mode nature of small volume PC cavities, which efficiently channels and recirculates radiation from embedded emitters into a single resonance.

In this work we describe our recent development of several devices: a quantum well ultra-fast PC laser with modulation rates in the $\mathrm{THz}$ at room temperature, ${ }^{6-8}$ a quantum dot (QD) laser with up to $30 \mathrm{GHz}$ modulation rates. ${ }^{9}$ and a $60 \mathrm{fJ}$ all optical modulator operating at $20 \mathrm{GHz}$ rates. ${ }^{10}$ In section 2 we review the fundamental properties of PC cavities and their applications to lasing. In section 3 we review the development of the $Q W$ laser. The QD PC laser is discussed in section 4, and in section 5 we review our work on modulators and propose a novel modulator based on free carrier depletion in cavity embedded quantum wells.

Further author information: (Send correspondence to J.V.: jela $\underline{a}$ stanford.edu)

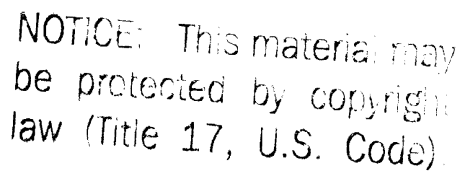




\section{PC PROPERTIES}

The photonic crystals used in our work were fabricated by standard electron beam lithography and reactive ion etching in Gallium Arsenide (GaAs) or Indium Phosplide (InP). Scanning electron micrographs of fabricated stuctures are shown in Fig.1. Cavity confinement results from total internal reflection in the vertical direction of the finite thickness slab (Fig.1(a)). and by distributed Bragg reflection due to the index contrast between air and semiconductor in the membrane plane. The lattice constant and radii of the periodic hole pattern are chosen for the particular wavelength operating range $(\approx 950 \mathrm{~nm}$ in this work). Cavities are created by removing and modifying holes and hole radii, as shown in Fig.1 for a square and triangular PC lattice. The optical volumes of these cavities are on the order of $V \approx(\lambda / n)^{3}$, and quality factors $\mathrm{Q}$ are in the rage of 1000 to 5000 . Thus, photons are recirculated for a long time and are confined very tightly, which affects the properties of emitters that are coupled to the cavity: In our work we use Indium Gallium Arsenide (InGaAs) quantum wells (QW) with 20\% In content, and Indium Arsenide (InAs) quantum dots (QD) grown in the middle of the membrane during sample fabrication. The radiative rate of an emitter coupled to a cavity can be enhanced or suppressed relative to the emission rate in bulk material. ${ }^{4}$ because the presence of the cavity modifies number of available radiative modes. For an emitter on resonance with the cavity. the enhancement is given by the Purcell factor F:

$$
F=\frac{1}{2} \frac{3}{4 \pi^{2}} \frac{Q}{V}\left(\frac{\lambda}{n}\right)^{3}
$$

Where the factor of $\frac{1}{2}$ accounts for emitter dipole orientation. F gives the radiation rate into the cavity mode relative to the emission rate into the bulk semiconductor. For small cavities only one cavity mode exists, and the overall fraction of light coupled to the cavity mode is given by $\beta=\frac{F}{f+F}$, where $f$ accounts for the reduction in the available states inside the photonic bandgap material. In photonic crystals considered in our work $F$ is on the order of 100 and $f$ can take on values between 0.1 and 1 depending on the emitter position: therefore the expected lower bound for $\beta$ is $99 \%$. A large $\mathrm{F}$ value leads to two outcomes: firstly, the spontaneous emission is enhanced due to a faster decay rate, and secondly, the stimulated emission is enhanced due to a high degree of coupling to the cavity mode and long storage times in the cavity. These two effects translate into a lower threshold power and short turn-on times.

For applications in high speed communication the laser power must be modulated quickly. Modulation can be achieved with large signal modulation by turning the laser on and off at a high rate, or via small signal modulation, where the laser is brought to threshold with a continuous signal and a small modulation takes the laser over threshold. Large values of $\beta$ result in large values of the relaxation oscillation frequency of the laser $\omega_{R}$, and enable small signal modulation at high rates with lower operating powers. ${ }^{12}$ Large signal modulation rates are ultimately limited by the carrier relaxation time from the excitation level to the lasing level. This limit can be reached at large $F$ values, which allow the cavity population to build up quickly by reducing the radiative lifetime of emitters.

\section{QUANTUM WELL PHOTONIC CRYSTAL LASERS}

Quantum wells are a well suited gain medium in PC cavities, because they can operate at room temperature and can be grown to overlap wit the maximum of the TE-like modes. Furthermore, the carrier capture time in quantum wells is on the order of picoseconds. and large modulation rates can be expected for PC cavities. We

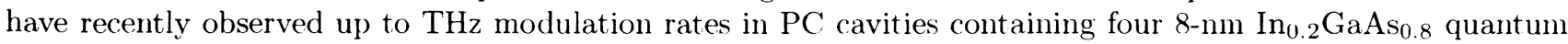
wells separated by 8 -nm of GaAs and embedded in a $172 \mathrm{~nm}$ thick membrane of GaAS. ${ }^{6}$ Square lattice cavities were fabricated in this sample, and lasing from single cavities (such as Fig.1(a)) and cavity arrays (such as Fig.1(b)) was investigated. The quantum wells were pumped above band with 3 ps pulses from a mode locked Ti:Sapphire laser with a repetition rate of $80 \mathrm{MHz}$ and a wavelength of $750 \mathrm{~nm}$. The laser chip was mounted in a He flow cryostat to control the operating temperature, and photoluminescence was collected with a confocal microscope setup (detailed in Ref. 4). Spectral resolution was obtained with a $75 \mathrm{~cm}$ spectrometer, and the time response was measured on a streak camera with a temporal resolution of $2 \mathrm{ps}$. The experiments were performed at a low temperature of $7 \mathrm{~K}$ for higher efficiency. and a high temperature of $100 \mathrm{~K}$ for faster modulation. The experimental results for the single cavity laser are reproduced in Fig.2. The cavity and QW spectrum are well 

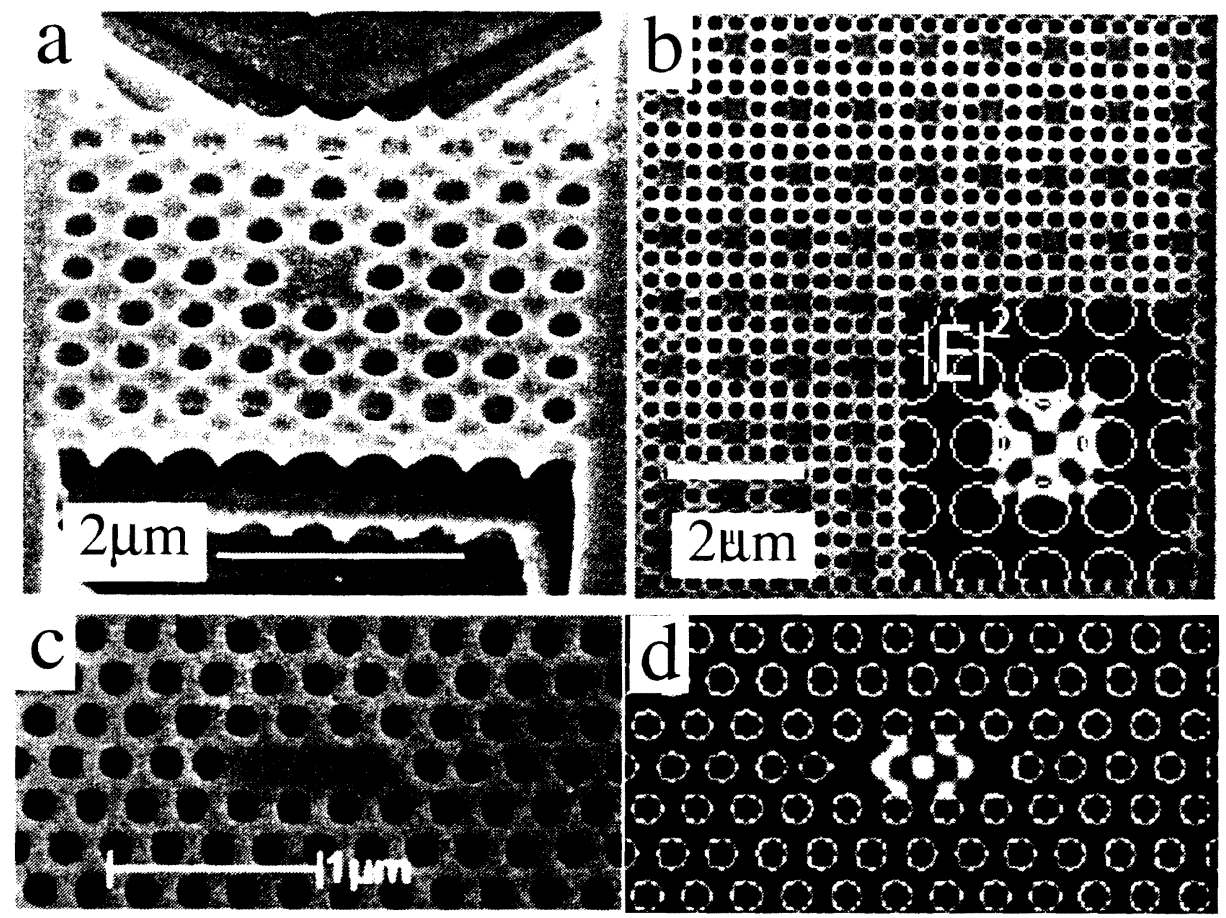

Figure 1. Scanning electron micrographs of photonic crystal cavities. (a) InP square lattice cavity with InGaAsP quantum wells for a PC laser operating at $1500 \mathrm{~nm}$ wavelengths shows the membrane structure. (b) GaAs square lattice cavity array with $\operatorname{In}_{0} .2 \mathrm{GaAs}_{0} .8$ quantum wells for operation at $950 \mathrm{~nm}$. The inset shows the amplitude squared of the Electric field of the cavity mode, which showcases the small optical volume $\left(\approx 0.55(\lambda / n)^{3}\right)$. (c) GaAs PC cavity with a triangular lattice. The active medium are InAs quantum dots operating at $940 \mathrm{~nm}$. (d) The field pattern of the fundamental mode of the triangular lattice cavity.

resolved in Fig.2(a). The laser threshold was measured to be approximately $6 \mu \mathrm{W}$ average power $(26 \mathrm{~mW}$ peak power) at $7 \mathrm{~K}$. The time resolved laser emission pulse is shown along with the excitation pulse in Fig.2(b) We observed a turn on delay of $\tau_{\text {delay }}=1.5 \mathrm{ps}$ at a temperature of $100 \mathrm{~K}$ and a delay of $3-4 \mathrm{ps}$ at $7 \mathrm{~K}$. The shorter delay at high temperature is due to a faster carrier scattering to the lowest excited states by phonon decay. The lasing pulse decayed with a lifetime of $2.13 \mathrm{ps}$. Large signal modulation was performed by a pulse train that was generated with an etalon inseted into the excitation beam path. Pulse separations of 15 ps and 9 ps are clearly visible in Fig.2(c), and show that this laser can be modulated at rates approaching the THz. Similar temporal profiles were observed when the multiple cavity laser was excited. The coupled cavity array exhibited a pulse lifetime of $2.18 \mathrm{ps}$ and a higher output power.

Although quantum wells are almost ideal for PC laser applications, they suffer from significant carrier recombination at exposed surfaces and defects. This recombination reduces the efficiency of the lasers and prevents operation at higher temperatures. In order to eliminate nonradiative carrier recombination, the laser was treated with a solution of $7 \% \mathrm{NH}_{4} \mathrm{~S}$. This treatment removes contanination and oxides from the GaAs and In0.2Ga0.8As surfaces and caps the fresh surface with sulfur atoms. ${ }^{8}$ The experimental results are shown in Fig. 3 . We observe a four fold reduction in lasing threshold after passivation (Fig.3(a)) due to a lower carrier loss to recombination. This efficiency translates into a faster photon buildup in the cavity, and slightly lowers the response time of the laser as shown in Fig.3(b). Because the surface treatment lifts off a small surface layer of oxidized GaAs, the wavelength of the cavities blue shifted and was accompanied by an increase in Q, most likely due to cleaning of the sample surface (Fig.3(c)).

The increase in carrier capture leads to more efficient laser performance that allows operation at room 

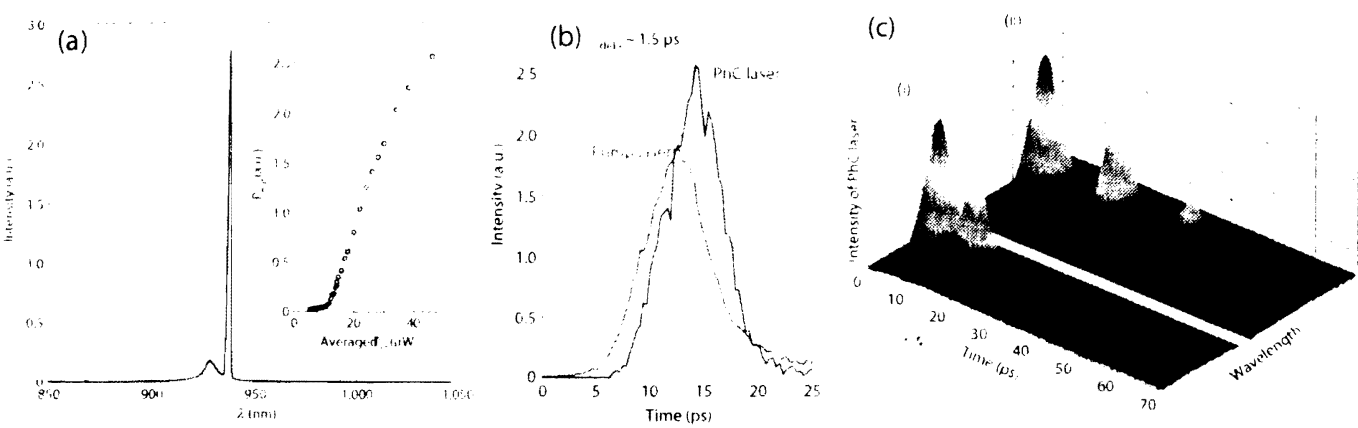

Figure 2. (a) Emission spectrum of a square lattice $I n_{0} .2 G a A s_{0} .8$ quantum well photonic crystal laser. The portion of the QW overlapping with the cavity is strongly amplified over the QW spectrum. The inset shows a power output versus power input curve with a threshold of $6 \mu \mathrm{W}$ of average pulse power $(26 \mathrm{~mW}$ peak power). (b) Response of the PC laser (blue) follows the pump (red) with a $1.5 \mathrm{ps}$ time delay at $100 \mathrm{~K}$. At $7 \mathrm{~K}$, the delay was $3-4$ ps. The laser pulse decay time is 2.13 ps. (c) Direct laser modulation with pulse separations of 15 ps (ii) and 9 ps (i). The pulse train was produced by an etalon, and the decay corresponds to attenuation of successive pump pulses.

temperature. ${ }^{\top}$ In Fig.4(a)(b) we show pulsed lasing from a passivated cavity at $10 \mathrm{~K}$ and $293 \mathrm{~K}$. Although the threshold increased significantly from $6.5 \mu \mathrm{W}$ at $10 \mathrm{~K}$ to $67 \mu \mathrm{W}$ average power $(290 \mathrm{~mW}$ peak power $)$ at room temperature and output power dropped, the lasing was stable. A much lower threshold was observed under continuous wave excitation. In Fig.4(c) we show CW operation at room temperature with a threshold of $9 \mu \mathrm{W}$. Phonon scattering is greatly increased at room temperature and leads to faster carrier capture times and carrier decay to the lasing level. In Fig.4(d), we show the drastic reduction in laser delay and pulse width at room temperature. The observed pulse rise and fall times were streak camera limited and allow us to extrapolate a modulation rate of $1 \mathrm{THz}$ for room temperature pulsed operation.

\section{QUANTUM DOT PHOTONIC CRYSTAL LASERS}

Thresholds in PC lasers can be further reduced by using quantum dots as the gain medium. Quantum dot based devices has a significantly lower active area and reduced surface recombination. As in a quantum well laser, the maximum modulation bandwidth is limited by the smaller of the relaxation oscillation frequency or the rate of carrier capture into the quantum dots. In conventional quantum dot lasers at low pump powers, the relaxation oscillation fre- quency is significantly smaller than the rate of carrier capture into the dots. This frequency can be enhanced by increasing the pump power. This technique was used to demonstrate small- signal modulation rates of several tens of gigahertz, ${ }^{13}$ but relatively large pump powers were necessary, making these lasers impractical for low-power applications. Our approach has been to use the large purcell enhancement and hence large $\beta$ factors to increase the modulation rate. ${ }^{9}$ We fabricated InAs quantum dot photonic crystal lasers in GaAs membranes (see structure in Fig.1(c)). We then investigated the large signal modulation response of these devices. We again used 3ps Ti:Sapphire pulses with a repetition rate of $80 \mathrm{MHz}$ as the pump. The pump wavelength was either aligned above the GaAs bandgap, or was tuned in resonance with one of the higher excited states of the quantun dots (p state). Time resolved data was taken with a streak camera, limited to $2 \mathrm{ps}$ temporal resolution. The experimental data is shown in Fig.5. In Fig.5(a) we show the power output versus power input curve for a quantum dot PC laser. We fit the experimental data (points), with a three level rate equation model given in Ref. 9. The best observed thresholds were on the order of $250 \mathrm{nW}$, with a typical value of $1 \mu \mathrm{W}$ average power. From our temporal measurements, we find that the maximum modulation rate is limited by the rate of carrier 


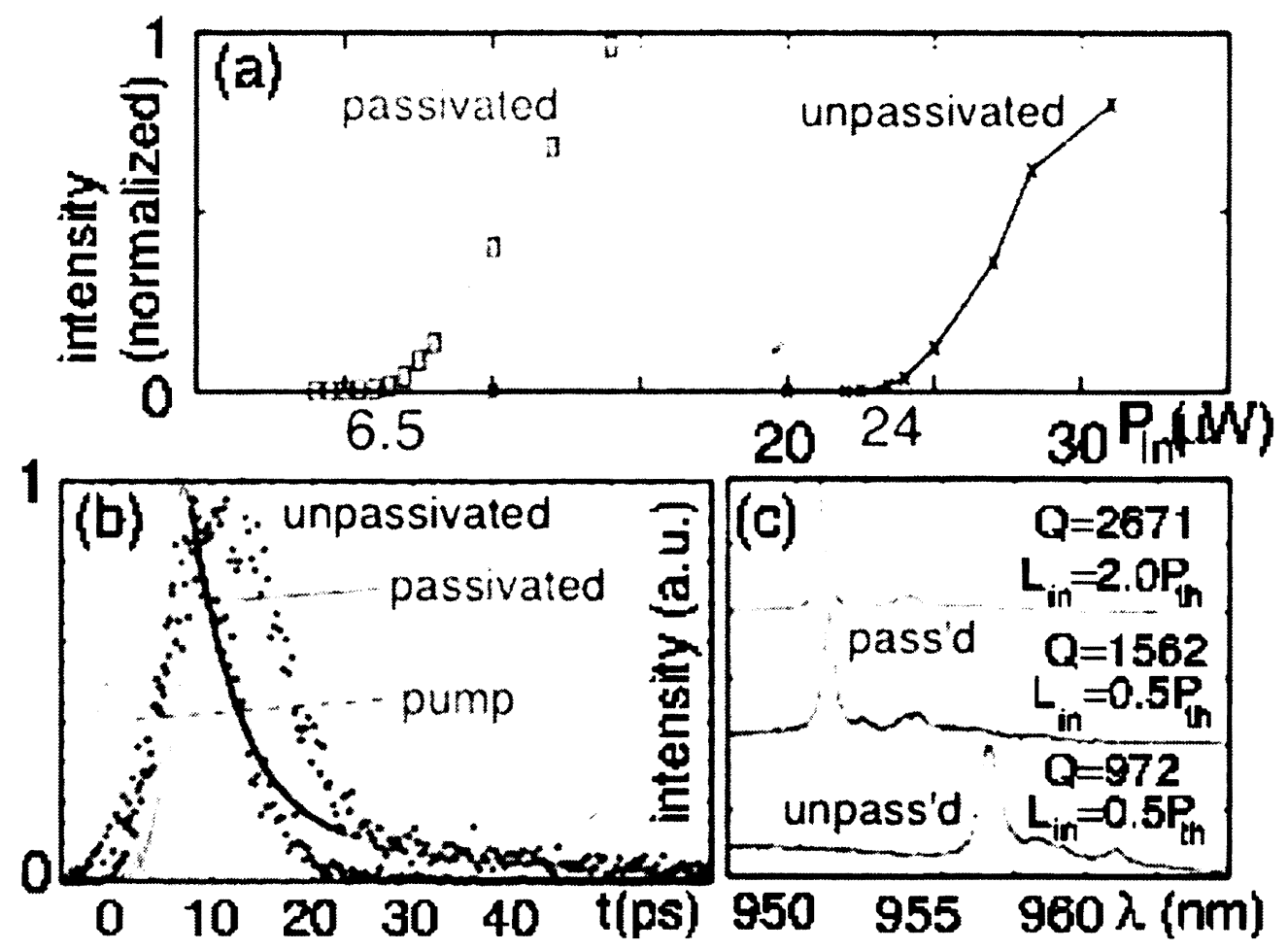

Figure 3. (a) Power-in power-out curve for the passivated and unpassivated QW PC: laser sample. The threshold drops from $24 \mu \mathrm{W}$ for the unpassivated sample to $6.5 \mu \mathrm{W}$ for the passivated sample. This indicates a four fold reduction in the nonradiative recombination rate. (b) Temporal response of the passivated and unpassivated laser at

capture into the dots, as expected. In Fig.5(b) we show the QD laser output pulse along with the pump pulse. A clear separation of approximately 13.5ps between the arrival of the pump pulse and the lasing pulse is observed. This time delay corresponds to the time it takes carriers to relax from high excited states of the quantum dot to the lowest lasing level. The time agrees well with rise times observed in time resolved photoluminescence of bulk quantum dots. Experiments performed at 10 and 15 times threshold indicate that the rise time is pinned at about 12 ps even at very high pump powers. From simulations we conclude that the rise time of quantum dot lasers is limited by the carrier capture time. However, in high- $\beta$ lasers this limit is practically achievable because it is approached at lower pump powers relative to threshold as opposed to quantum dot lasers not employing stong cavity effects where higher power pumping is needed. Above threshold, higher pump powers lead to faster de- cay times due to increased stimulated emission rates. Small- mode volume PC cavities can be used to achieve large pho- ton densities and speed up this process. Fig.5(c) shows the laser response at various pump powers, demonstrating the reduction in decay time with increasing pump power. We observed a minimum decay time of 8.5 ps at pump powers around five times threshold Fig.5(a). For higher pump powers the laser response appears largely unchanged. We attribute this to large carrier densities causing the gain to saturate, preventing further decrease of the decay time, but more work is necessary to characterize saturation effects in our quantum dots. Based on the measured laser response and the results of simulations, we predict that our laser can be modulated at large-signal modulation rates approaching $30 \mathrm{GHz}$.

\section{PHOTONIC CRYSTAL OPTICAL MODULATOR}

The PC laser benefits not only from the high Q and low optical volume, but also from the fast carrier recombination, which contributes to the fast laser modulation rate by sweeping out residual carriers. We have exploited this effect to realize a fast PC based optical modulator. The modulation results from a free carrier induced refractive index shift, which changes the transmission window of a PC cavity. Because of the small cavity size 
(a) $10 \mathrm{~K}$,pulsed

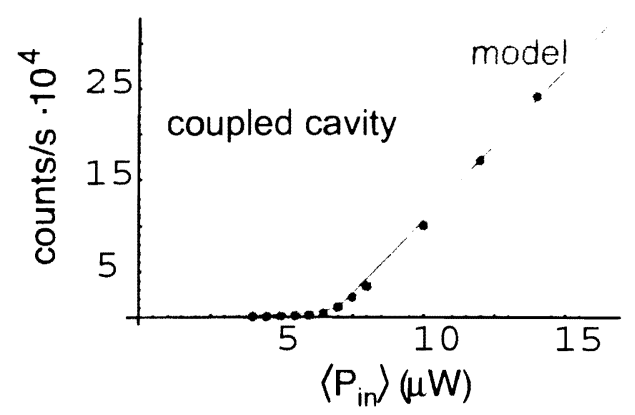

(c) $10 \mathrm{~K}, \mathrm{CW}$

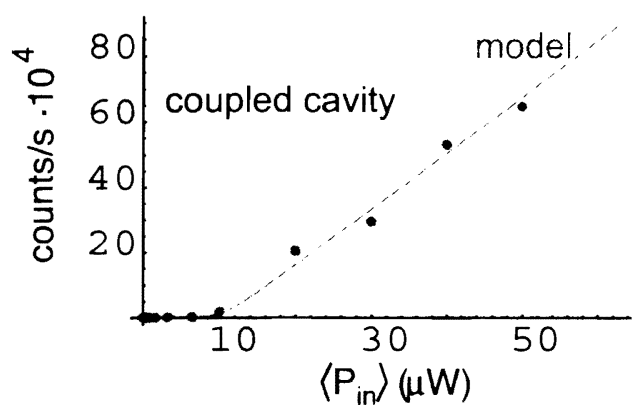

(b) 293K,pulsed
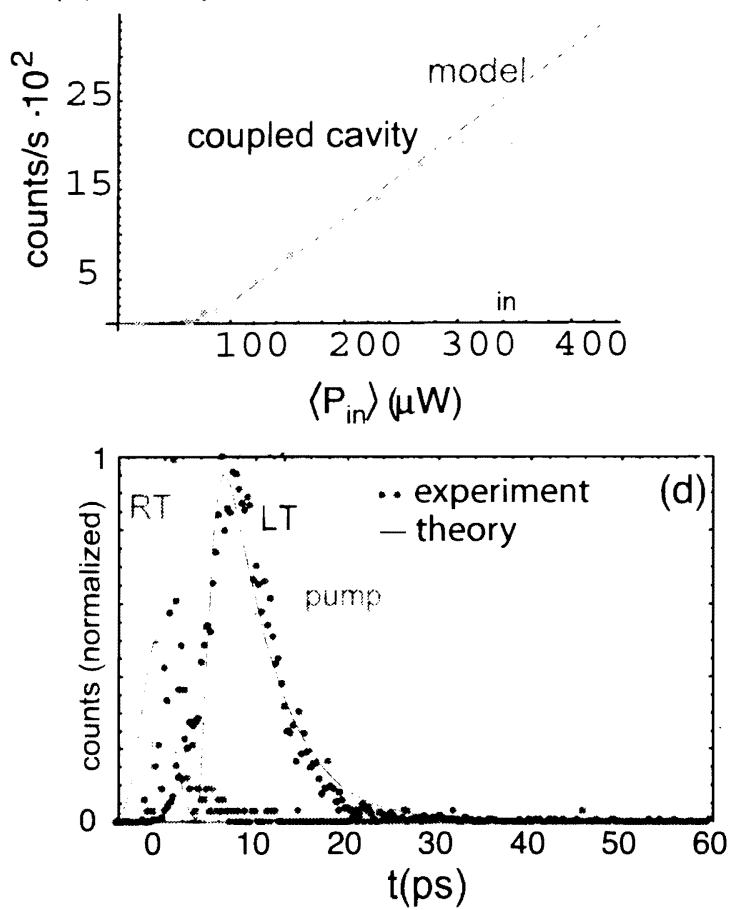

Figure 4. (a) Power-in power-out curve for a passivated coupled cavity quantum well laser array at $10 \mathrm{~K}$ under pulsed excitation. A $6.5 \mu \mathrm{W}$ threshold is observed. (b) At room temperature, the threshold increases approximately ten fold, but pulsed lasing persists. (c) Continuous wave operation at $10 \mathrm{~K}$ with a threshold of $9 \mu \mathrm{W}$. (d) Effect of temperature on laser response time. At room temperature carrier capture and depletion processes are enhanced by phonon scattering, and allow $\mathrm{THz}$ modulation rates.

and high Q, small powers are needed to change the index appreciably. The PC based optical modulator benefits mostly from enhanced carrier recombination at surfaces of PC holes.

In Fig.6(a,b), we show the tuning response of a PC cavity which is pulsed above band with a 3 ps Ti:Sapphire pulse. In Fig.6(a), a streak camera image shows the wavelength as a function of time. The presence of free carriers causes the index to shift with the arrival of the pump pulse at to and detunes the cavity resonance. The time to return to the steady state cavity resonance is measured $50 \mathrm{ps}$. In Fig.6(b) we plot the cavity resonance at $\mathrm{t} 0$ and $\mathrm{t} 0+50 \mathrm{ps}$, which shows the 1/e suppression at the two times. In Fig.6(c) and (d) similar data is shown for a three-hole defect cavity in a quantum well sample. The quantum cavity exhibited lasing threshold at $180 \mathrm{fJ}$ pulse energy (15 microwatt average pump power). In Fig.6(c), the time response of the pulsed cavity at threshold is shown. The decay time is $7.5 \mathrm{ps}$, and corresponds to the return time of the cavity to the un-shifted resonance. As shown in Fig.6(d), the suppression is also 1/e of the value.

In our earlier work, ${ }^{10}$ switching occurred as an above-band optical pulse injected free carriers into a PC cavity in a GaAs membrane containing InAs quantum dots. The presence of free carriers locally altered the refractive index of the material, and with it, the cavity resonance, as shown in Figure 8. The free carrier lifetime, which is mostly determined by the surface recombination rate. is strongly modified by the presence of holes in the PC membrane, and can theoretically be as low as $10 \mathrm{ps}$. Our published experimental results show a 50 ps lifetime of carriers in our sample, but unpublished results came close to the 10-20 ps range.

Switching speeds can be further increased in a quantum well based device, as shown in Fig.6(c.d). This switching occurs in a PC laser operating close to threshold, where the lasing action in addition to the surface recombination depletes injected carriers and changes the refractive index of the semiconductor. We have measured such switching rates with optical pumping. and found that they are on the order of $7.5 \mathrm{ps}$, as shown in Fig.6(c). 
(a)

(b)
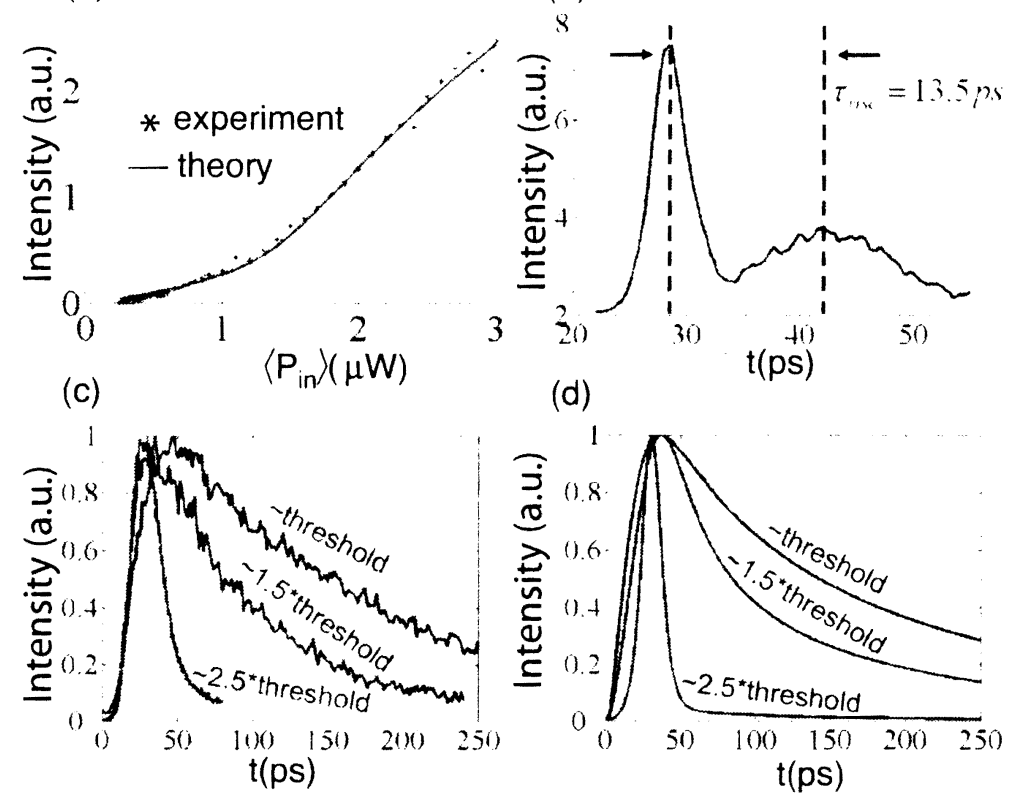

Figure 5. (a) Power-in power-out curve for the quantum dot PC laser, with a threshold at $1 \mu \mathrm{W}$. (b) Carrier capture rate of 13.5ps leads to a turn-on delay of the quantum dot laser. (c) Experimentally observed power dependence of the laser modulation rate, along with the theoretical model (d). At large powers, the modulation rate is limited by the capture time.

Such switches can be employed to quickly modulate a strong $\mathrm{CW}$ laser in order to obtain fast pulses with large powers, which are important for long distance communication.

\section{CONCLUSION:}

We have demonstrated a variety of ultra-compact. ultrafast optical elements that can drive the next wave of information processing devices. We have shown that photonic crystals allow an unprecedented degree of control over the radiative properties of cavity-coupled emitters. We have exploited this property to realize high Purcell factors and therby high $\beta$ values. High $\beta$ values have led to exceptionally low thresholds extremely high modulation rates for quantum well and quantum dot lasers. Furthermore, we have shown that surface passivation of quantum well lasers can be used to increase their efficiency by a factor of four, and allow us to realize room temperature continuous wave lasing and $\mathrm{THz}$ modulation rates. Finally, we have also demonstrated an optical modulator based on the free-carrier induced refractive index change. This modulator operates with $20 \mathrm{GHz}$ rates and switching energies as low as $60 \mathrm{fJ}$. The mode of operation can be realized in a quantum well PC modulator, where the switching times are reduced to $7.5 \mathrm{ps}$, because carrier recombination is enhanced in the quantum well.

\subsection{Acknowledgments}

This work has been supported by the MARCO IFC Center, NSF Grants ECS-0424080 and ECS-0421483. D.E. was sup- ported by the NDSEG and NSF fellowships, and I.F. by the NDSEG fellowship. We also thank Pierre Petroff and Nick Stoltz of UC Santa Barbara, as well as YoshihisaYamamoto and Bingyang Zhang of Stanford University, for quantum dot growth. 

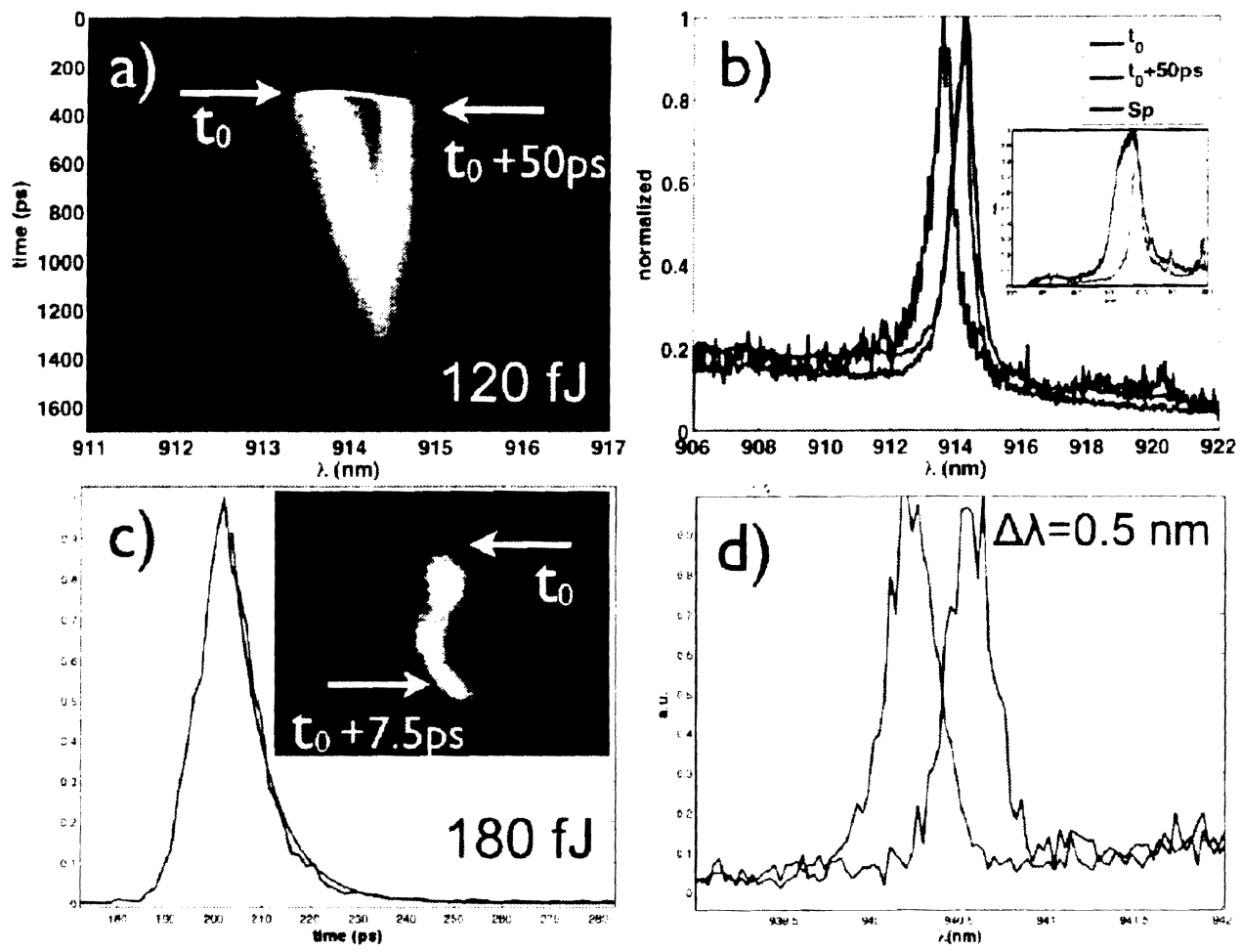

Figure 6. (a) Free carrier based optical switch in a GaAs photonic crystal cavity. Above band pulses from a Ti:Sapphire laser inject free carriers and modify the refractive index of the GaAs cavity. The change in index decays with the carrier decay time, which is reduced due to the high surface area to volume ratio on photonic crystals. In (b) we show the cavity resonance at the pulse arrival $\left(\mathrm{t}_{0}\right)$ and $50 \mathrm{ps}$ later. The cavity resonance shifts by approximately a linewidth, giving $1 / \mathrm{e}$ attenuation with a 50ps switching rate. (c) Quantum well modulator based on the free carrier refractive index change. A quantum well PC laser was operated at threshold. The lasing action and large carrier recombination in quantum wells depletes carriers within approximately 7.5 ps with a $1 /$ e suppression in (d).

\subsection{References}

\section{REFERENCES}

1. D. Miller, "Rationale and Challenges for Optical Interconnects to Electronic Chips," Proc. of the IEEE 88(6), pp. 728-749, 2000.

2. M. Soljacic and J. Joannopoulos, "Enhancement of Nonlinear Effects Using Photonic Crystals," Nature Materials 3. pp. 211-219, 2004.

3. M. Lee and P. Fauchet, "Two-dimensional silicon photonic crystal based biosensing platform for protein detection," Optics Express 15(8), pp. 4530-4535, 2007.

4. D. Englund, D. Fattal, E. Waks, G. Solomon, B. Zhang, T. Nakaoka, Y. Arakawa, Y. Yamamoto, and J. Vučković, "Controlling the Spontaneous Emission Rate of Single Quantum Dots in a Two-Dimensional Photonic Crystal," Physical Review Letters 95. p. 013904, July 2005.

5. D. Englund, A. Faraon. I. Fushman, N. Stoltz. P. Petroff, and J. Vučković, "Controlling cavity reflectivity with a single quant um dot," Nature 450(6), pp. 857-61. 2007.

6. H. Altug. D. Englund. and J. Vučković. "Ultrafast photonic crystal nanocavity laser," Nature Physics 2, pp. $484-488,2006$.

7. D. Englund, H. Altug. I. Fushman. and J. Vučković, "Efficient Terahertz Room-Temperature Photonic Crystal Nanocavity Laser." Appl. Phys. Lett. 91. p. 071126, July 2007.

8. D. Englund, H. Altug. and J. Vučković. "Low-Threshold Surface-Passivated Photonic Crystal Nanocavity" Laser," Appl. Phys. Lett. 91, p. 071124, July 2007. 
9. B. Ellis, I. Fushman, D. Englund. B. Zhang. Y. Yamannoto, and J. Vuckoric, "Dynamics of Quantum Dot Photonic Crystal Lasers." Appl. Phys. Lett. 90. p. 151102, June 2007.

10. I. Fushnnan, E. Waks, D. Englund. N. Stoltz. P. Petroff, and J. Vučković. "Ultrafast nonlinear optical tuning of photonic crystal cavities." Applied Physics Letters $90(9)$, p. 091118. 2007.

11. H. Altug and J. Vučkorić. "Photonic crystal nanocavity array laser." Opt. Express 13(22). pp). 8819 882x. 2005.

12. G. Bjork and Y. Yannanoto, "Analysis of semiconductor microcavity lasers using rate equations," IEEE Journal of Quantum Electonics 27: pp. 2386-96. Nov. 1991.

13. S. Fathpour, Z. Mi, and P. Bhattacharya, "High-speed quantum dot lasers ", Jourmal of Physics D 38. p. $2103,2005$.

\author{
NoTlCE Ths materia may \\ be woree hy copuright \\ law Tite 17, u.S Code).
}

